\title{
Les traumatismes de la verge : à propos de 23 cas
}

\author{
Yaya SOW, Papa Ahmed FALL, Babacar DIAO, Boubacar FALL, Alain Khassim NDOYE, \\ Baye Assane DIAGNE
}

Service d'Urologie-Andrologie, Centre Hospitalier Universitaire A. Le Dantec Dakar, Sénégal

\begin{abstract}
RESUME
Les auteurs rapportent, à partir d'une étude rétrospective, 23 cas de traumatismes du pénis reçus dans leur pratique quotidienne urologique au Sénégal.

Les différents cas se répartissaient comme suit : fracture de verge (rupture des corps caverneux) (19 cas), lésion des corps caverneux par arme à feu ( 2 cas) dont un cas associé à une lésion urétrale, rupture de la veine dorsale du pénis (1 cas) et dilacération du fourreau pénien (1 cas).

L'âge moyen des patients était de 32,4 ans avec des extrêmes de 19 ans et 47 ans. La prise en charge chirurgicale précoce des ruptures des corps caverneux a permis de réduire le risque de complications.
\end{abstract}

Mots clés : traumatisme, verge, corps caverneux

\section{INTRODUCTION}

Le traumatisme du pénis est une pathologie rare. La lésion la plus fréquente est la rupture des corps caverneux au décours d'un traumatisme de la verge en érection. Les amputations et les strangulations sont surtout observées chez les patients psychiatriques et constituent un phénomène exceptionnel. Le but de cette étude était de rapporter les cas de traumatisme du pénis pris en charge au service d'Urologie Andrologie de l'hôpital Aristide Le Dantec et d'en préciser les aspects étiologiques, cliniques et thérapeutiques.

\section{PATIENTS ET MÉTHODES}

A partir d'une étude descriptive, nous avons rapporté 23 cas de traumatisme de la verge reçus en milieu hospitalier urologique. Cette étude s'est intéressée aux dossiers des patients présentant un traumatisme de la verge et admis de janvier 2000 à janvier 2007. Pour chaque patient nous avons étudié :

- l'état civil : âge, situation matrimoniale;

- les aspects cliniques : délai de consultation, mécanisme du traumatisme, résultats de l'examen clinique, et les aspects para cliniques ;

- les aspects thérapeutiques : délai de prise en charge, caractéristiques per-opératoires de la lésion et le geste thérapeutique ;

- les résultats thérapeutiques avec comme critères d'appréciation : l'aspect esthétique, l'existence ou non de plaque de fibrose, la qualité de l'érection, des rapports sexuels et de la miction.

\section{RESULTATS}

Nous avons répertorié 23 cas. L'âge moyen était de 32,4 ans avec des extrêmes de 19 ans et 47 ans (Figure 1).

Le délai moyen entre le traumatisme et la consultation en milieu urologique était de 1,4 jour avec des extrêmes allant de 1 heure à 11 jours. Ainsi $82,6 \%$ des patients avaient consulté avant la 24 ème heure. Seuls deux patients étaient reçus tardivement après le 10 ème jour.

Les différents cas se répartissaient comme suit : 19 cas de fracture de verge (rupture des corps caverneux) (Figure 2), 2 cas de lésion des corps caverneux par arme à feu dont un cas associé à une lésion urétrale, 1 cas de rupture de la veine dorsale du pénis, et 1 cas de dilacération du fourreau pénien (Figure 3).

Le mécanisme du traumatisme en cause était dominé par le faux pas du coït $(n=8)$ et la réintroduction brutale et forcée du pénis en érection dans la culotte $(n=8)$ (Tableau 1).

\section{Correspondance :}

Dr Yaya SOW - Service d'Urologie, Hopital Aristide Le Dantec, BP 3001 Dakar, Sénégal - Tel +221 77540923 Email yahyasowdj@yahoo.fr 


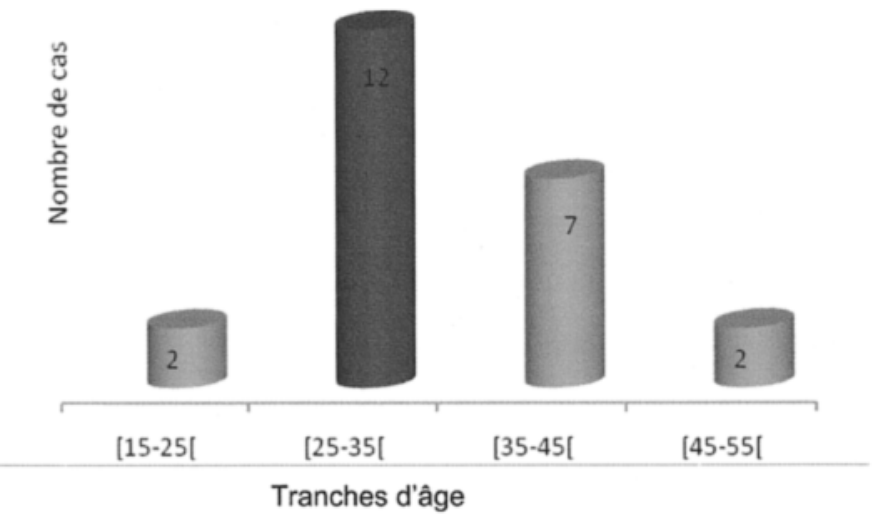

Figure 1 : Répartition par tranches d’âge.

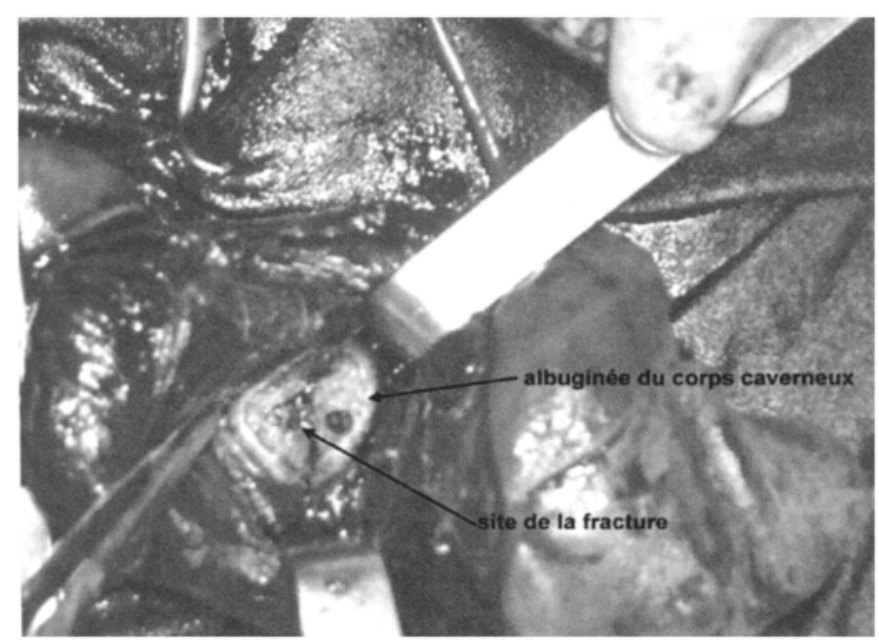

Tableau 1 : Répartition en fonction du mécanisme du traumatisme.

\begin{tabular}{lc} 
Mécanisme lésionnel & Nombre de cas \\
\hline Masturbation & 3 \\
Faux pas du coït & 8 \\
$\begin{array}{l}\text { Réintroduction brutale et forcée de } \\
\text { la verge en érection }\end{array}$ & 8 \\
Choc sur bord du lit & 1 \\
Lésion par arme à feu & 2 \\
Lésion par couteau après tentative & 1 \\
d'auto circoncision & \\
\hline
\end{tabular}

Figure 2 : Rupture de l'albuginée d'un corps caverneux
droit : vue per-opératoire.

Figure 2 : Rupture de l'a
droit : vue per-opératoire.

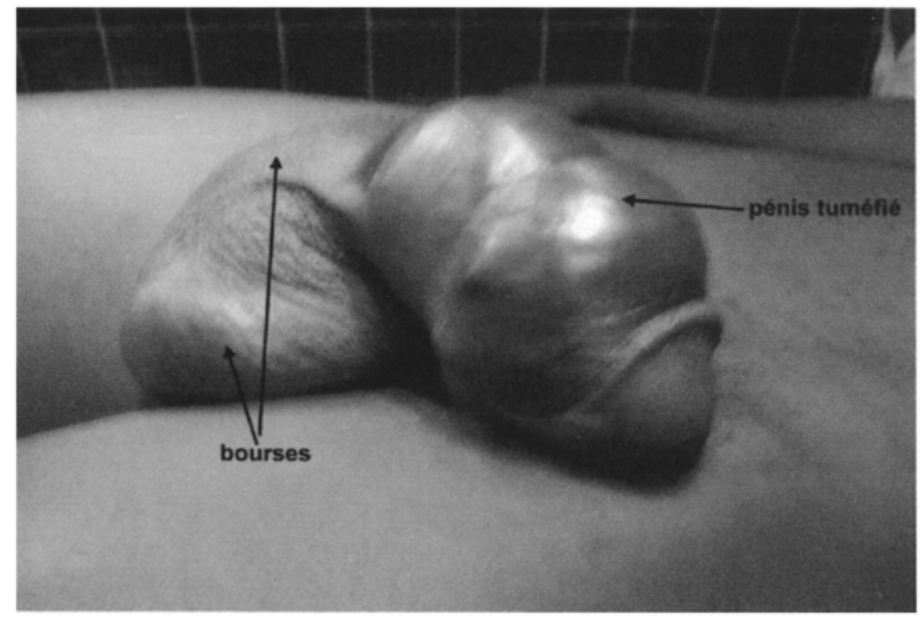

Figure 3 : Dilacération du fourreau pénien après tentative d'auto circoncision. 
Pour les cas de traumatisme par arme à feu, il s'agissait d'une manœuvre accidentelle. L'un des patients ayant appuyé accidentellement sur la gâchette de son pistolet artisanal camouflé sous sa ceinture, tandis que pour l'autre les circonstances étaient imprécises.

La symptomatologie clinique était typique et évocatrice dans la presque totalité des cas de fracture de verge (Figure 4). Cependant, aucun cas d'urétrorragie ou de dysurie n'a été noté. Les examens complémentaires (échographie, cavernographie) ont été réalisés dans un cas.

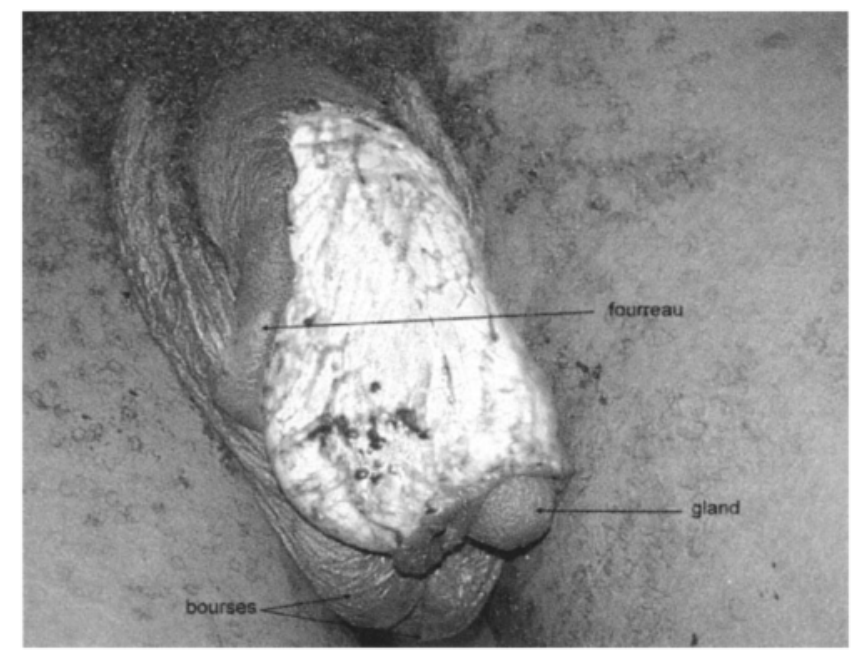

Figure 4 : Aspect typique d'une fracture de verge avec œdème important et déviation de la verge : vue préopératoire.

La rupture des corps caverneux intéressait le coté droit dans la plupart des cas (11 cas). Elle siégeait au niveau du tiers moyen ( 11 cas) et au tiers proximal ( 8 cas), alors que le tiers distal n'était pas impliqué. La longueur moyenne de la lésion des corps caverneux était de 1,9 centimètre avec des extrêmes de 1 et 3 centimètres. Les deux cas de lésions par arme à feu étaient représentés par une plaie de la face dorsale de la verge associée à une rupture de l'albuginée des corps caverneux. Dans un des cas, il existait une rupture partielle de l'urètre pénien.

Le traitement des fractures de verge a consisté, dans la majorité des cas après abord circonférentiel, en une évacuation de l'hématome suivie d'une albuginéorraphie.

Dans le cas de rupture de la veine dorsale superficielle, une évacuation de l'hématome et une ligature de cette veine étaient réalisées.

Les lésions par arme à feu ont bénéficié d'un parage associé à une albuginéorraphie et urétrorraphie dans le cas où il y avait une rupture partielle de l'urètre.

La dilacération de la verge était traitée par un parage et une circoncision. Du fait de l'élasticité du fourreau, il n'y a eu aucune difficulté pour la fermeture cutanée.

La durée moyenne d'hospitalisation était de 1,25 jour avec des extrêmes de 1 et 4 jours.
Les résultats thérapeutiques globaux étaient satisfaisants dans $78,3 \%$ des cas. Cependant, chez 5 cas de fracture de verge, il existait des complications à type de dysérection (1 cas), de douleurs péniennes per-coïtales ( 2 cas) et de plaques fibreuses au niveau du corps caverneux avec coudure modérée de la verge lors de l'érection associées à des douleurs per-coïtales ( 2 cas). Les deux cas de lésions péniennes par arme à feu ont évolué favorablement. Avec un recul moyen de plus de 18 mois, aucun cas de sténose urétrale ou de dysfonctionnement érectile n'était noté.

\section{DISCUSSION}

\section{Fracture de verge}

C'est une affection rare. Cependant l'incidence est probablement sous estimée. La pudeur et l'ignorance poussent certains patients à ne pas consulter. Sylla et al. [18] en 2000, après une revue de la littérature, n'avaient relevé que 628 cas, alors que selon Eke [4], le nombre total de cas décrits dans la littérature entre 1935 et 2001 est de 1642. La plupart des publications concernent des séries limitées ou de simples rapports de cas.

II s'agit principalement d'une pathologie du sujet jeune. Plusieurs autres auteurs $[4,15,20]$ retrouvent les mêmes constatations. Pour Eke [4], cela s'explique par une plus grande vigueur des rapports sexuels à cet âge. Dans la série d'Ishikama et al. [10], la majorité des patients est âgée de 20 à 50 ans, âge auquel d'après eux, les rapports sexuels sont en principe beaucoup plus fréquents.

Pour les fractures de verge, le faux pas du coït est l'étiologie la plus fréquemment rapportée dans la littérature. Elle est secondaire à un rapport sexuel vigoureux ou acrobatique [18]. Le pénis en érection vient percuter la symphyse pubienne, le périnée, la face interne de la cuisse ou la fesse de la partenaire lorsqu'il glisse hors du vagin $[8,18]$. Ces fractures de verges peuvent également être dues à des manipulations de la verge en érection dans le but de stopper une érection matinale [5] ou de la cacher en réintroduisant brutalement le pénis. Ce dernier facteur est surtout observé dans les pays en voie de développement où les conditions socioéconomiques imposent la cohabitation, la promiscuité et obligent parfois le sujet à essayer de camoufler une érection matinale [18]. La masturbation a été également fréquemment citée $[18,20]$ mais, du fait de la gêne du patient, cette étiologie est rarement avouée. Plus exceptionnellement, des cas de rupture des corps caverneux ont été notés sur une verge flaccide [5].

Le délai moyen de consultation est de 39 heures $(1,6$ jour). Cependant ce délai moyen est en général inférieur à 24 heures [7]. Il est de 21,5 heures dans la série de Zargooshi [20]. II a aussi été rapporté des cas de fractures de verges vues au stade de séquelles à type de troubles de l'érection ou de coudure du pénis ou de noyaux fibreux des corps caverneux [15].

Le diagnostic est essentiellement clinique : la symptomatologie le plus souvent évocatrice associe :

- douleur aiguë du pénis contemporaine d'un craquement bien décrit par le patient et souvent entendu par la partenaire [10] ; 
- détumescence rapide du pénis [8] ;

- hématome qui débute en regard de la lésion, dont l'extension est déterminée par l'intégrité ou non du fascia profond du pénis donnant l'aspect du pénis en aubergine [15] ;

- déviation du pénis en aval de la lésion et controlatérale à celle-ci.

La palpation d'un défect de l'albuginée est souvent possible [15], surtout quand l'hématome n'est pas trop important. Le signe du roulement ou "Rolling sign" est un signe qui permet d'identifier le site de la fracture : le caillot qui se forme au niveau de la fracture, qui est palpable sous la peau du pénis, roule en dessous de cette dernière [20].

D'autres signes peuvent être présents, surtout quand une rupture de l'urètre est associée. L'urètrorragie est alors le signe le plus constant et le plus précoce. La symptomatologie peut cependant être fruste ou le patient peut être vu au stade tardif pour coudure de la verge ou pour plaques de fibrose au niveau des corps caverneux [7].

Les examens complémentaires sont le plus souvent inutiles car le diagnostic est souvent posé dès la clinique. Ceci explique que dans notre série un seul cas ait bénéficié d'explorations complémentaires. Néanmoins, dans les formes frustres de fracture de verge, les cas vus tardivement ou lorsque une rupture de l'urètre est suspectẻe, ces examens peuvent être utiles permettant de préciser siège exact de la fracture $[8,20]$.

La cavernographie permet en cas de doute sur l'atteinte de l'albuginée de visualiser et de localiser précisément la brèche [15].

L'échographie pénienne est l'examen de première intention car non invasif et peu coûteux. Elle permet de préciser le siège de la rupture et la taille de l'hématome $[8,18]$.

L'imagerie par résonance magnétique (IRM) a une sensibilité supérieure à celle de l'échographie pénienne dans le diagnostic de la rupture des corps caverneux. Elle peut préciser une éventuelle lésion urétrale associée [19]. II s'agit néanmoins d'un examen coûteux et qui peut retarder la prise en charge chirurgicale, ce qui rend son utilisation en pratique courante difficile [15].

L'urètro-cystographie rétrograde permet de rechercher une lésion urétrale associée. Certains la préconisent systématiquement [6] tandis que d'autres ne la réalisent qu'en cas de signes évocateurs d'une atteinte urétrale [20].

Dans notre série, la rupture des corps caverneux siégeait le plus souvent sur le coté droit et au niveau du tiers moyen. D'autres auteurs rapportent le siège unilatéral droit de la lésion sans que cela ne puisse être expliqué [20]. Par contre la situation au niveau du tiers proximal du corps caverneux est le plus souvent notée $[10,20]$.

La longueur de la fracture variable, était en moyenne de 1,9 $\mathrm{cm}$ et variait entre 1 et $2,5 \mathrm{~cm}$ dans la série de Fergany [6].

L'association à une rupture de l'urètre est rare. La lésion de l'urètre est estimée à 1,3\% dans la série de Zargooshi [20].
Elle est le plus souvent publiée dans le cadre de rapports de cas [19], ou mise dans la rubrique des traumatismes de l'urètre antérieur. Elle constitue la principale lésion associée à rechercher et s'observe volontiers en cas de fracture bilatérale des corps caverneux [7]. Elle est beaucoup plus fréquente en cas de lésions en rapport avec un faux pas du coït $[6,20]$. Cela est probablement lié à la violence du traumatisme dans ce cas.

Dans certains cas, la fracture de la verge peut être confondue avec une rupture de la veine dorsale du pénis du fait du contexte similaire mais la symptomatologie est en générale plus fruste. Cette pathologie est encore beaucoup moins fréquente que la rupture des corps caverneux (1 cas dans notre série). Sharma [16] rapporte aussi un cas tandis que Cavalcanti et al. [2] en ont noté quatre. Le diagnostic est en général peropératoire [16]. Elle peut être suspectée après avoir éliminé une fracture de verge par les examens complémentaires [15].

La classique controverse qui existait dans les anciennes publications [9] quant au choix entre les traitements médical et chirurgical n'est plus d'actualité. Le traitement chirurgical précoce est de nos jours largement utilisé et conseillé par la plupart des auteurs $[7,8,15]$. Ce traitement présente des avantages certains tels que la réduction de la durée d'hospitalisation et de la fréquence des complications $[8,18]$.

La voie d'abord circonférentielle a été préférée dans notre série, de même que pour certains auteurs $[8,10,19]$, car elle laisse une cicatrice esthétique surtout chez nos patients qui étaient tous déjà circoncis. En plus, dans ce contexte d'urgence, la situation exacte des lésions était difficile à préciser en préopératoire. Cette voie permet alors de faire un bilan complet des lésions. D'autres trouvent cette voie excessive et pourvoyeuse d'infections, d'œdèmes et de nécrose cutanée [4].

La voie élective, hémi circonférentielle ou longitudinale, en regard de la lésion est réservée aux ruptures de siège proximal en l'absence d'autres lésions [15]. Elle impose la connaissance du siège exact de la rupture. Elle est moins délabrante mais laisse souvent une cicatrice inesthétique [7].

La réparation des lésions caverneuses est effectuée après évacuation de l'hématome et hémostase soigneuse [8]. La suture de l'albuginée est effectuée par des points simples séparés de fils résorbables $[7,15]$. Certains ont proposé l'utilisation de patch (vaginal, peau, greffon veineux) en cas de défect important [3].

La lésion associée de l'urètre est, dans ce contexte, le plus souvent réparée par une urétrorraphie termino-terminale dans le même temps opératoire $[2,15,19]$.

Après traitement chirurgical précoce, nous avons obtenu $75 \%$ de bons résultats. La plupart des auteurs s'accordent sur le fait que la réparation chirurgicale est moins pourvoyeuse de complications $[8,10,15]$. Le taux de complications dans les traitements conservateurs varie de 10 à $53 \%$ et comporte notamment un risque d'érections douloureuses, d'hématomes persistants pouvant s'infecter et évoluer vers l'abcédation, de fistules artério-veineuses, de dysfonction érectile et de ruptures urétrales méconnues [6]. Cependant pour Mydlo et al. [14], 
sur cinq patients qui avaient refusé la chirurgie, quatre avaient une fonction érectile normale et un seul présentait une courbure du pénis séquellaire après traitement médical.

\section{Les automutilations sexuelles}

La strangulation est un phénomène rare ; nous n'avons noté aucun cas dans notre série. Soixante-dix huit cas sont rapportés dans la littérature depuis 1971 [15].

L'amputation de la verge quant à elle est extrêmement rare. Environ 100 cas sont identifiés dans la littérature [17]. Stunell et al. [17] rapportent un cas d'automutilation chez un psychotique qui s'était amputé les testicules et le pénis avec un couteau. De même, Moufid et al. [13] rapportent deux cas d'automutilation de la verge dont un cas d'amputation de la verge et un cas de strangulation par un anneau métallique. La lacération du fourreau pénien n'a pas été retrouvée dans la littérature.

La strangulation de la verge est souvent secondaire à la mise en place volontaire d'objets métalliques circulaires (anneau, écrou, roulement à bille...) ou non métalliques (élastique, ficelle et même cheveu), à la base de la verge [15]. Tandis que pour l'amputation pénienne plusieurs instruments sont utilisés : lame de rasoir, couteau de cuisine ou paire de ciseaux [17].

Les automutilations sont surtout observées chez les patients psychiatriques, les transsexuels et chez les sujets sous l'emprise de l'alcool $[13,15]$. D'autres, par contre, évoquent des croyances religieuses ou culturelles complexes [17].

Pour la strangulation de la verge, le principal problème posé est surtout la difficulté d'ablation de l'agent d'étranglement. Plusieurs méthodes sont décrites : la ponction de l'œdème et de la congestion veineuse d'aval et la méthode d'enroulement d'un fil, comme pour ôter une bague d'un doigt. Cette dernière consiste à enrouler un fil sur le pénis en aval de l'anneau d'étranglement dans le but de réduire le calibre du pénis. Elle est préconisée dans les lésions précoces. Cependant tous ces moyens peuvent s'avérer inefficaces et l'utilisation d'une meule ou d'une scie à métaux est parfois le seul recours [15].

Quant à l'amputation de la verge, la réimplantation du pénis doit être tentée à chaque fois que cela est possible [15]. Les résultats de cette réimplantation ont été améliorés par la microchirurgie qui autorise des réimplantations jusqu'à 24 heures d'ischémie [11]. Cependant, avant toute réimplantation, un avis psychiatrique est indispensable car un psychotique chronique récidivera sûrement. Aussi, en l'absence de microscope, la technique préconisée par Moufid et al. [13] peut être tentée. Celle-ci consiste à réparer uniquement les corps caverneux et l'urètre après avoir dénudé le pénis et l'avoir enfoui dans le scrotum pour éviter la nécrose cutanée. Un deuxième temps de plastie cutanée est alors nécessaire. En cas d'impossibilité de réimplantation de la verge, la régularisation du moignon avec méatoplastie est le dernier recours permettant d'assurer un confort mictionnel.

\section{Lésions péniennes par armes à feu}

Le mécanisme lésionnel en matière de plaies par arme à feu permet de distinguer les plaies de pratique civile des plaies de guerre. Dans notre série, il s'agissait essentiellement de plaies de pratique civile accidentelles. L'hémorragie est le principal motif de consultation. En cas de lésion urétrale, on peut noter une urétrorragie. Les lésions par balle imposent la recherche d'un orifice d'entrée et de sortie afin de préciser la présence ou non du projectile.

Les lésions par arme à feu sont variables. Il peut s'agir d'une atteinte des corps caverneux et ou de l'urètre parfois associée à une atteinte testiculaire [12].

Les lésions péniennes par arme à feu posent surtout un problème infectieux lié au caractère ouvert de la lésion et au corps étranger constitué par le projectile. Le parage de la plaie, l'ablation du corps étranger éventuel et la suture des corps caverneux sont préconisés [1, 12]. La lésion urétrale quant à elle peut être réparée en même temps, mais le risque infectieux fait préférer à certains un traitement en deux temps selon la technique de Bengt Johanson [1].

\section{CONCLUSION}

La gravité des traumatismes du pénis réside dans la répercussion sur les fonctions sexuelle et urinaire. La dysfonction érectile est la principale hantise des patients en cas de fracture des corps caverneux.

La prise en charge précoce de ces patients a fortement réduit l'incidence des complications. La prise en charge des automutilations impose un avis psychiatrique pour prévenir les récidives.

\section{REFERENCES}

1. BORKI K., OUKHEIRA H., DRAOUI M.I. : Les plaies de l'urètre par projectiles de guerre. A propos de 19 cas. Acta Urol. Belgica, 1987, $55: 625-635$.

2. CAVALCANTIA.G., KRAMBECK R., ARAÚJOA. et al. : Management of urethral lesions in penile blunt trauma. Int. J. Urol., 2006, 13 : 1218-1220.

3. CENDRON M., WHITMORE K.E., CARPINIELLO V. et al. : Traumatic rupture of the corpus cavernosum : evaluation and management. J. Urol., 1990, 144 : 987-991.

4. EKE N. : Fracture of the penis. Br. J. Urol., $2002: 89: 555-565$.

5. EL-SHERIF A.E., DAULEH M., ALLOWNEH N., VIJAYAN P. : Management of fracture of the penis in Qatar. Br. J. Urol., 1991; 68 :622-625.

6. FERGANYA.F., ANGERMEIER K.W., MONTAGUE D.K. : Review of Cleveland Clinic experience with penile fracture. Urology, 1999, 54 : 352-355.

7. GRIMAF., PAPAREL P., DEVONEC M., PERRIN P., CAILLOT J.L., RUFFIONA. : Prise en charge des traumatismes des corps caverneux du pénis. Prog. Urol., 2006, 16 : 12-18.

8. HINEV A. : Fracture of the penis : treatment and complications. Acta Med. Okayama, 2000, $54: 211-216$.

9. HIRASAWA S., TSUBOI N., ABE H. et al. : Fracture of the penis : Report of 10 cases and a review of 231 cases in Japan. Hinyokika kiyo, 1983, 29 : 1047-1052.

10. ISHIKAWA T., FUJISAWA M., TAMADA H., INOUE T., SHIMATANI $\mathrm{N}$. : Fracture of the penis : nine cases with evaluation of reported cases in Japan. Int. J. Urol., 2003, $10: 257-260$.

11. JORDAN G.H., GILBERT D.A. : Management of amputation injuries of the male genitalia. Urol. Clin. North Am., 1989, $16: 359-367$.

12. MIANNE D., GUILLOTREAU J., SERGENT H. : Les plaies périnéales en pratique de guerre. Ann. Urol., 1997, 31 : 303-308. 
13. MOUFID K., JOUAL A., DEBBAGH A., BENNANI S., EL MRINI M. : L'automutilation génitale : à propos de 3 cas. Prog. Urol., 2004, 14 : 540-543.

14. MYDLO J.H., HAYYERI M., MACCHIA R.J. : Urethrography and cavernosography imaging in a small series of penile fractures : a comparison with surgical findings. Urology, 1998, $51: 616-619$.

15. PRUNET D., BOUCHOT O. : Les traumatismes du pénis. Prog. Urol., 1996, 6 : 987-993.

16. SHARMA G.R. : Rupture of the superficial dorsal vein of the penis. Int. J. Urol., 2005 12: 1071-1073.

17. STUNELL H., POWER R.E., FLOYD M. Jnr., QUINLAN D.M. : Genital self-mutilation. Int. J. Urol., 200613 : 1358-1360.

18. SYLLA C., DIALLO A.B., FALL P.A. et al. : Fracture de verge : à propos de 12 cas. Andrologie, 2000, $10: 407-411$.

19. TOUITI D., AMEURA., BEDDOUCHA., OUKHEIRA H. : La rupture de l'urètre au cours des fractures de la verge : A propos de 2 observations. Prog. Urol., 2000, $10: 465-468$.

20. ZARGOOSHI J. : Penile fracture in Kermanshah, Iran : report of 172 cases. J. Urol., 2000, $164:$ 364-366.

Manuscrit reçu : Juin 2008 ; accepté Juillet 2008.

\author{
ABSTRACT \\ Penile trauma: A study of 23 cases \\ Yaya SOW, Papa Ahmed FALL, Babacar DIAO, \\ Boubacar FALL, Alain Khassim NDOYE, \\ Baye Assane DIAGNE
}

The authors report 23 cases of penile injuries based on a retrospective study of their urological practice in Senegal.

The various cases were distributed as follows: fracture of the penis (19 cases), corpora cavernosa and urethral gunshot injuries (2 cases), rupture of the superficial dorsal vein of the penis ( 1 case) and laceration of the penile skin (1 case). The mean age of these patients was 32.4 years.

Early surgical treatment of all penile fractures reduces the complication rate.

Key words: trauma, penis, corpora cavernosa 